\title{
5GINFIRE: Enabling an NFV Based Experimentation of Vertical Industries in the 5G Context
}

\author{
Flávio de Oliveira Silva ${ }^{1}$, Pedro Frosi Rosa ${ }^{1}$, Halid Hrasnica ${ }^{2}$, Anastasius Gavras ${ }^{2}$ \\ ${ }^{1}$ Faculty of Computing (FACOM) - Federal University of Uberlandia (UFU) \\ Uberlandia - MG - Brazil \\ ${ }^{2}$ Eurescom GmbH \\ Heidelberg - Germany \\ \{flavio,pfrosi\}@ufu.br, \{hrasnica, gavras\}@eurescom.eu
}

\begin{abstract}
G$ aims to provide a wireless access network that supports the requirements of different vertical markets such as automotive and smart cities. $5 G$ oriented services use the Internet of Things (IoT) and will use the network programmability by integrating Software-defined Networking (NFV) and Network Function Virtualization (NFV). This context presents several multi-facet challenges to experiment with these services. 5GINFIRE is a $5 G$ oriented experimental facility where different actors of the $5 G$ ecosystem can experimentally contribute to $5 G$ development and deployment. This work presents the $5 G G I N$ FIRE ecosystem, highlights some of its capabilities and some $5 G$ ongoing experiments.
\end{abstract}

\section{Introduction}

At this moment the world is engaged in the design and deployment of the next generation of International Mobile Telecommunications (IMT-2020), a.k.a, 5G [Gupta and Jha 2015] [Agiwal et al. 2016].

5G aims to provide a wireless access network that supports the requirements of different vertical markets such as agriculture, automotive, energy, health, media and entertainment, public safety, manufacturing, and smart cities [Zafeiropoulos et al. 2018]. The $5 \mathrm{G}$ network will provide not only an enhanced Mobile Broadband (eMMB), but UltraReliable Low-Latency Communication (URLLC) and Massive Machine Type Communication (mMTC) [Elayoubi et al. 2016] [Lema et al. 2017].

The Internet of Things (IoT) is a common technology used on these different verticals. IoT is an enabler for a completely new set of services, applications, and business models to all the different actors in the telecommunication ecosystem.

$5 \mathrm{G}$ also will rely on cloud computing and in the network programmability by integrating Software-defined Networking (NFV) and Network Function Virtualization (NFV).

One characteristic of these verticals is that they do not operate and evolve in a silo mode anymore, relying on closed and proprietary technological environments, but they are becoming open ecosystem built on top of shared physical infrastructures, (re)using the same open source components and APIs and sharing resources utilizing virtualization.

This context presents a number of multi-facet challenges (such as interoperability, inter-connectivity, federation, resource sharing, slicing, resource scheduling, operations) 
of unprecedented magnitude and complexity and it calls for the appropriate identification, introduction, integration and efficient operation of a series of common architectural elements and infrastructure assets that should be combined together with the vertical-specific components.

The 5GINFIRE project goal is to provide a $5 \mathrm{G}$ oriented experimental facility where different actors of the $5 \mathrm{G}$ ecosystem (startups, industry, SDOs, operators, academia, research institutes) can experimentally contribute to $5 \mathrm{G}$ development and deployment.

5GINGRE infrastructure enables the experimentation of Experimental Vertical Instances (EVIs), a composition of several virtual functions spanning all layers from application and services to networking with a focus on $5 \mathrm{G}$ verticals.

This work presents the 5GGINFIRE ecosystem, highlights some of its capabilities and some $5 \mathrm{G}$ ongoing experiments.

This work is organized as follows: Section 2 describes the 5GINFIRE facility. Section 3 details some 5G related experiments that uses 5GINFIRE. Section 4 presents some final considerations and presents the future directions of this research.

\section{The 5GINFIRE Ecosystem}

The 5GINFIRE concept focuses on the Management and Orchestration (MANO) side of the $5 \mathrm{G}$ network that is composed of a set of Virtual Functions of Network and Verticals (VxFs). In this context, a network slice is an end-to-end logical mobile network with the $\mathrm{VxFs}$ deployed on the different cloud infrastructures managed by an operator.

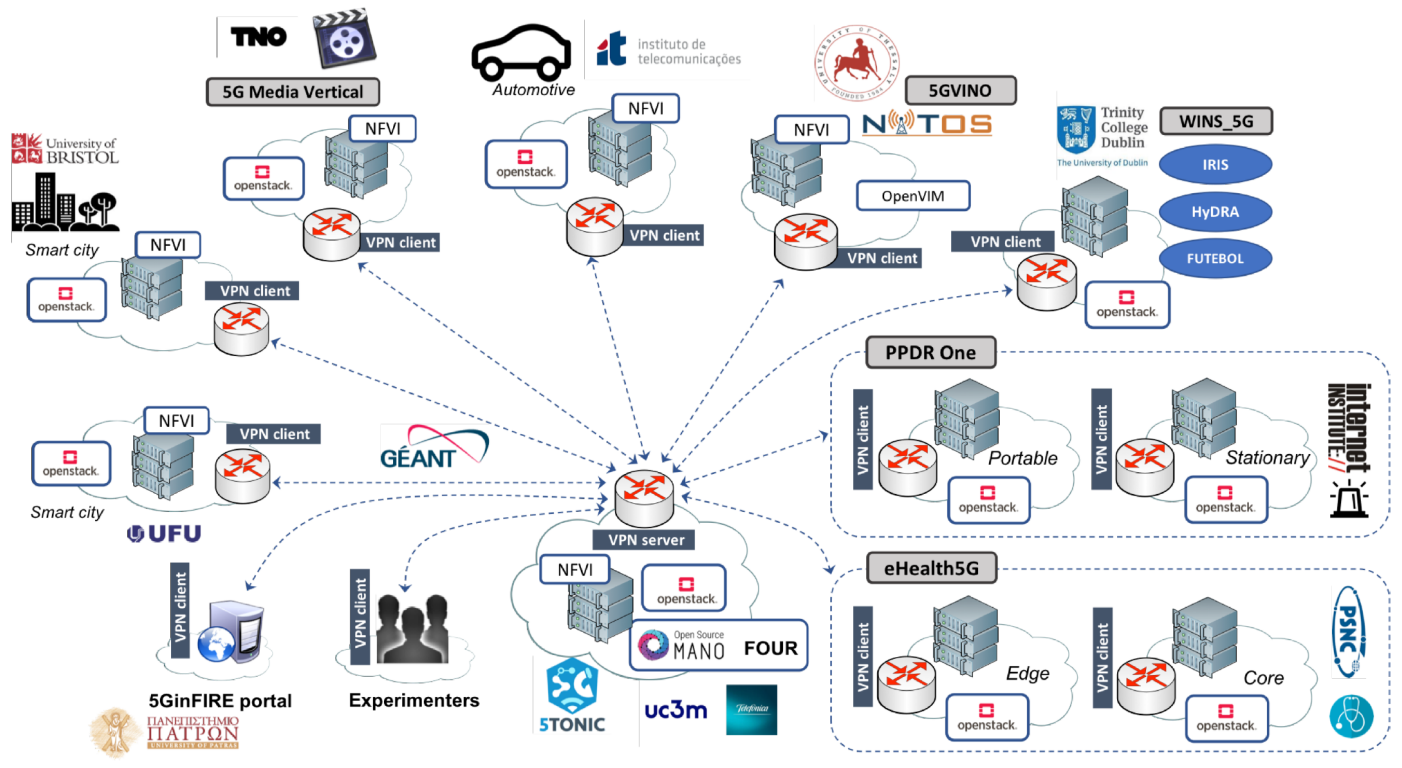

Figure 1. Overview of The 5GINFIRE Core MANO Platform

The 5GINFIRE ecosystem brought a series of particular challenges for the MANO platform to be used, especially related to the high diversity of functions to be considered and the multi-domain nature of the infrastructure to be managed. 
The technical solution adopted by the project considers the utilization of a single orchestration domain, where an NFV orchestrator, implemented with Open Source MANO (OSM) [ETSI 2018], manages and coordinates the creation of Network Services (NS). A Network Service is as a composition VxFs. Each of these VxFs may be in turn deployed at any of the experimental infrastructures made available by 5GinFIRE partners, called infrastructure providers.

The experimenter creates one or more NS and using the 5GINFIRE portal [5GINFIRE 2018] is requests the deployment over the 5GinFIRE infrastructure and then it can run the $5 \mathrm{G}$ related experiments. The University of Patras (UoP), in Greece, hosts the 5GINIRE portal.

Figure 1 presents an overview of the 5GINFIRE Core MANO platform. The OSM release four [Hoban et al. 2018] is deployed in the 5G Telefonica Open Innovation Laboratory (5TONIC) [IMDEA 2016] made available by Telefónica Investigación y Desarrollo(TID) and Universidad Carlos III de Madrid (UC3M). This instance of OSM orchestrates the VxFs across all infrastructure providers as expressed by the NS. Considering a $5 \mathrm{G}$ network, it represents the MANO instance of a network operator.

Each infrastructure provider is in charge of the deployment and maintenance of a Virtualized Infrastructure Manager (VIM), compliant with the OSM software stack. On top of that, the NFV orchestrator of 5GinFIRE, deployed at 5TONIC, interacts with the VIMs of the testbed providers involved in a service deployment: it coordinates the allocation and setup of the computing, storage and network resources which are necessary for the instantiation and interconnection of the VxFs that compose the network service.

Besides the VIM, each infrastructure provider explores one of the $5 \mathrm{G}$ verticals. To this end, it also has a local deployment of different sensors and equipment to support specific services of a vertical.

At the Instituto de Telecomunicações in Aveiro (ITAv) the focus is the automotive vertical. The infrastructure made available by the University of Bristol (UNIVBRIS), supports experimentation activities over a smart city environment in the city of Bristol (UK). The experimentation facility at the Federal University of Uberlândia (UFU), located at Uberlândia (Brazil), enables trials with smart city applications with a particular consideration on the edge network resources.

The infrastructure provided by the NITOS testbed, hosted by the University of Thessaly (Greece), provides access to programmable resources for wireless networking, SDN and cloud computing facilities. The extension of the NITOS testbed was called 5 GVINO.

A reconfigurable radio testbed at Trinity College Dublin (Ireland), called IRIS, supports radio hardware, cloud-RAN, NFV, and SDN technologies. This testbed has been extended and made available for experimentation activities in 5GinFIRE. We refer to this testbed extension as $\mathrm{WINS}_{5} G$.

In the the Poznan Supercomputing and Networking Center (PSNC), in Polandm, an eHealth experimental vertical facility, called eHealth5G supports experimentation in the area of telemedicine and eHealth, offering access to: realistic eHealth equipment; a small Edge Cloud, close to eHealth devices; and a core cloud accessible via MPLS/Optical 
service provider network.

NITOS and IRIS tested were created inside the Future Internet Research and Experimentation (FIRE) [Gavras et al. 2007]. Considering the number of assets already deployed on Future Internet Research and Experimentation (FIRE) testbeds, one of the goals of 5GINFIRE is to reuse these assets by enabling the deployment of the NFV network services atop of the FIRE based facilities. NITOS and IRIS testbeds are concrete examples of this reuse.

By using open calls, new infrastructure providers are engaging in the 5GINFIRE ecosystem, integrating new facilities. The TNO institute, in the Netherlands, will host the 5G Media Vertical, a testbed facility that targets the 5G Media Vertical industry. The Internet Institute (II), in Slovenia, hosts the Public Protection and Disaster Relief (PPDR) facility for outdoor and indoor experiments with $5 \mathrm{G}$ network architectures and services in this area.

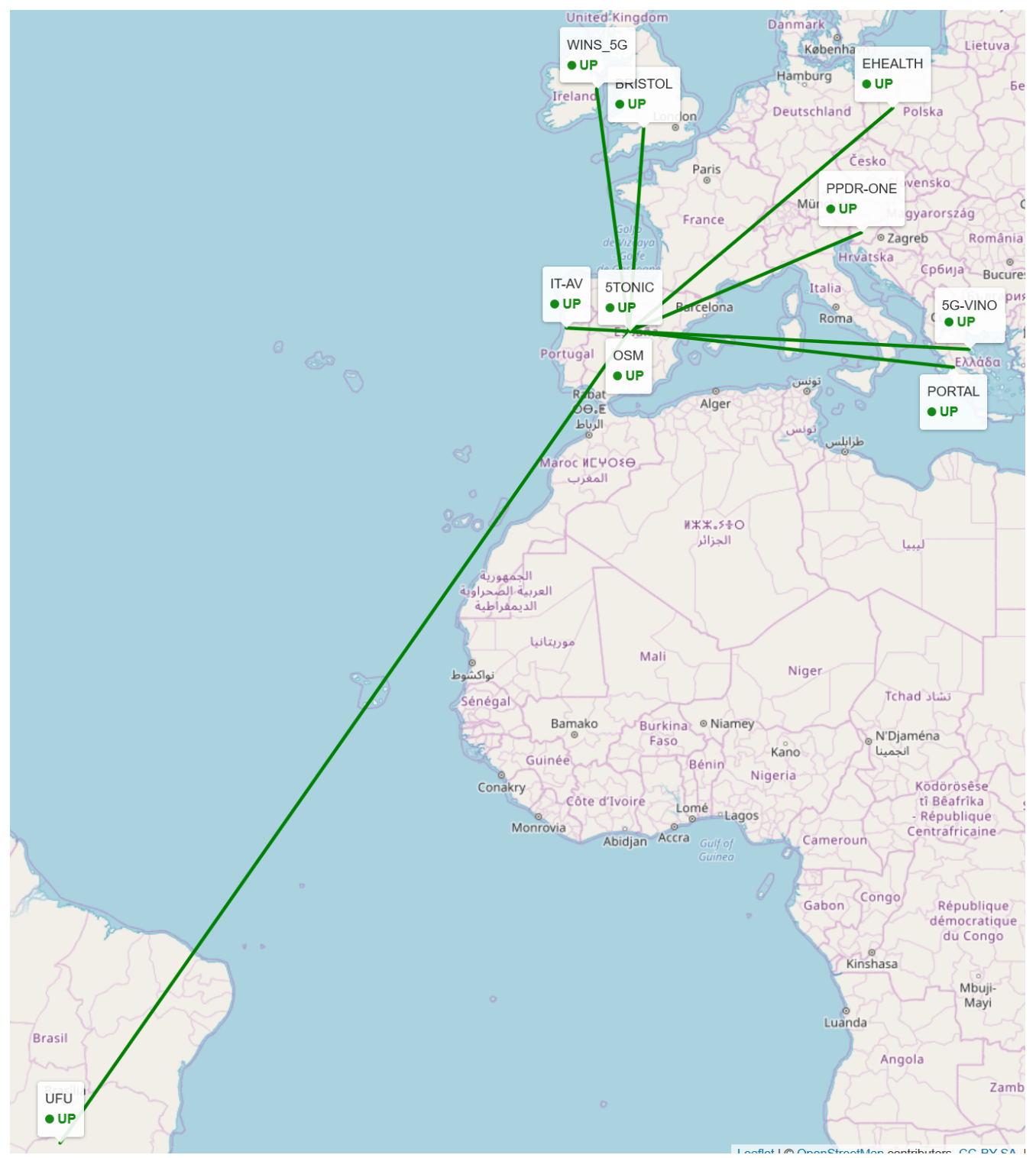

Figure 2. 5GINFIRE Facility Map 
5GINFIRE spans between Europe and Brazil. Figure 2 presents the facility map provided in the health check system that monitors the different infrastructure providers already deployed and interconnected.

\section{5GINFIRE Experiments}

Under the 5GINFIRE several experiments are ongoing at this moment are they are related to different verticals such as automotive and smart cities [5GINFIRE 2018].

The Context-Aware Video Controller for autonomous transport and security monitoring (CAVICO) allows controlling the video stream from the onboard camera in the function of transmission QoS and video QoE parameters measured by specific software components. The Hybrid Communications to Foster 5G Vehicular Services (SURROGATES) aims at virtualizing regular On-Board Unit (OBU) tasks requiring a high computing load, based on a hybrid communication system that allows a proactive connection between the real OBU and a virtual one.

The Vulnerable Road Users Safety (VRU-Safe) is an experimental evaluation of a network service with computing and networking capabilities, which is able to operate in a distributed manner utilizing either MEC, or Cloud capabilities (in the form of virtualized network functions), depending on the location and the network information of the involved OBUs and VRUs.

The Service Function Chaining orchestration application for low latency guarantees (SFCLola) provides tenants with a latency-aware service function chaining management while minimizing support required from infrastructure operators.

The 5G Smart City Robotic Surveillance Platform (RobotView5G) enables realtime video surveillance using $\mathrm{WiFi}$ and mobile networks from remote-controlled robots, drones, and other video-monitoring devices and conduct experiments with this scenario.

These are a few examples of different experiments conducted in the 5GINFIRE ecosystem. At this moment, other ones related to eHealth, mission-critical services and entertainment verticals are also taking place.

\section{Concluding Remark and Future Work}

$5 \mathrm{G}$ oriented services will use the Internet of Things (IoT) by connecting different devices located in the network edge. These services also will use a programmable network based on Software-defined Networking (NFV) and Network Function Virtualization (NFV). The experimentation of such services are complex and pose several challenges.

The 5GINFIRE facility enables an NFV based experimentation of different services focusing on several vertical industries.

With an interconnected facility that spans over Europe and Brazil, the 5GINFIRE testbed supports at this moment several experiments in the automotive and smart cities scenarios.

The 5GINFIRE facility is still evolving and soon will support the 5G media vertical and mission critical in PPDR scenarios, thus improving the experimentation of 5G oriented services contributing to $5 \mathrm{G}$ design and deployment. 


\section{References}

5GINFIRE (2018). 5ginfire Experiments.

Agiwal, M., Roy, A., and Saxena, N. (2016). Next Generation 5g Wireless Networks: A Comprehensive Survey. IEEE Communications Surveys Tutorials, 18(3):1617-1655.

Elayoubi, S. E., Fallgren, M., Spapis, P., Zimmermann, G., Martín-Sacristán, D., Yang, C., Jeux, S., Agyapong, P., Campoy, L., Qi, Y., and Singh, S. (2016). 5g service requirements and operational use cases: Analysis and METIS II vision. In 2016 European Conference on Networks and Communications (EuCNC), pages 158-162.

ETSI (2018). Open Source MANO (ETSI).

Gavras, A., Karila, A., Fdida, S., May, M., and Potts, M. (2007). Future Internet Research and Experimentation: The FIRE Initiative. SIGCOMM Comput. Commun. Rev., 37(3):89-92.

Gupta, A. and Jha, R. K. (2015). A Survey of 5g Network: Architecture and Emerging Technologies. IEEE Access, 3:1206-1232.

Hoban, A., Israel, A., Sepúlveda, A. T., Salguero, F. J. R., de Blas, G. G., Kashalkar, K., Ceppi, M., Shuttleworth, M., Harper, M., Marchetti, M., Velandy, R., Almagia, S., and Little, V. (2018). OSM Release Four Technical Overview.

IMDEA (2016). 5tonic.

Lema, M. A., Laya, A., Mahmoodi, T., Cuevas, M., Sachs, J., Markendahl, J., and Dohler, M. (2017). Business Case and Technology Analysis for 5g Low Latency Applications. IEEE Access, 5:5917-5935.

Zafeiropoulos, A., Gouvas, P., Fotopoulou, E., Tsiolis, G., Xirofotos, T., Bonnet, J., Carrozzo, G., Rizou, S., Gavras, A., Barros, M. J., Costa-Perez, X., Prasad, A., Gramaglia, M., Tzanakaki, A., Simeonidou, D., Cosmas, J., Fallgren, M., Muñoz, R., and Vilalta, R. (2018). Enabling Vertical Industries Adoption of 5g Technologies: A Cartography of Evolving Solutions. In 2018 European Conference on Networks and Communications $(E u C N C)$, pages 1-9. 